\title{
Author Index to Volume 11
}

URBAN DESIGN International (2007) 12, 65. doi:10.1057/palgrave.udi.9000185

Allan A 173

Bagaeen SG 117

Biddulph M 49

Brand D 99

Cobo, A 213

Echols SP 203
Gehl J 29

Hadjri K 3

Hayward R 1, 69, 131

Hooper A 49

Kaefer LJ 29

Nassar HF 203
O'connor Z 21

Oliveira V 187

Pinho P 187

Primerano F 173

Punter J 49

Reigstad S 29
Sintusingha S 151

Soltani A173

Somenahalli S 173

Talen 83

Zhang D 133

\section{Keyword Index to Volume 11}

activity 173

$\mathrm{Al}$ Ain 3

alternative future scenario method 151

American planning history 83

awards 49

Bangkok superblocks 151

brownfield sites 117

Cairo 203

colonial cities

(Australia and

New Zealand) 99

community

involvement 117

competitions 49

courtyard design 133

courtyard housing 133 developing cities 151

digital technology 21

ecosystems 203

environmental colour mapping 21

evaluation 49

façade colour 21

facades 29

ground floors 29

housing 49

interaction 29

Jane Jacobs 71

Lisbon 187 military land conversion 117

morphological periods 187

neighbourhood 173

new urbanism 83

Nile 203

Oporto 187

people 29

Portugal 187

preference 49

public space 29

residential 49

space syntax 3

spatial 3

sustainability 151 sustainable development 117 sustainable urban form 133

travel 173

UAE 3

urban 3

urban design 173

urban design 99

urban history 99

urban morphology 187

urban renewal 133

urban representation 99

urban sprawl 151

vernacular architecture 133

walkability 173

water 203 